\title{
Dilated cardiomyopathy in thyrotoxicosis
}

\author{
S Goland, S Shimoni, O Kracoff
}

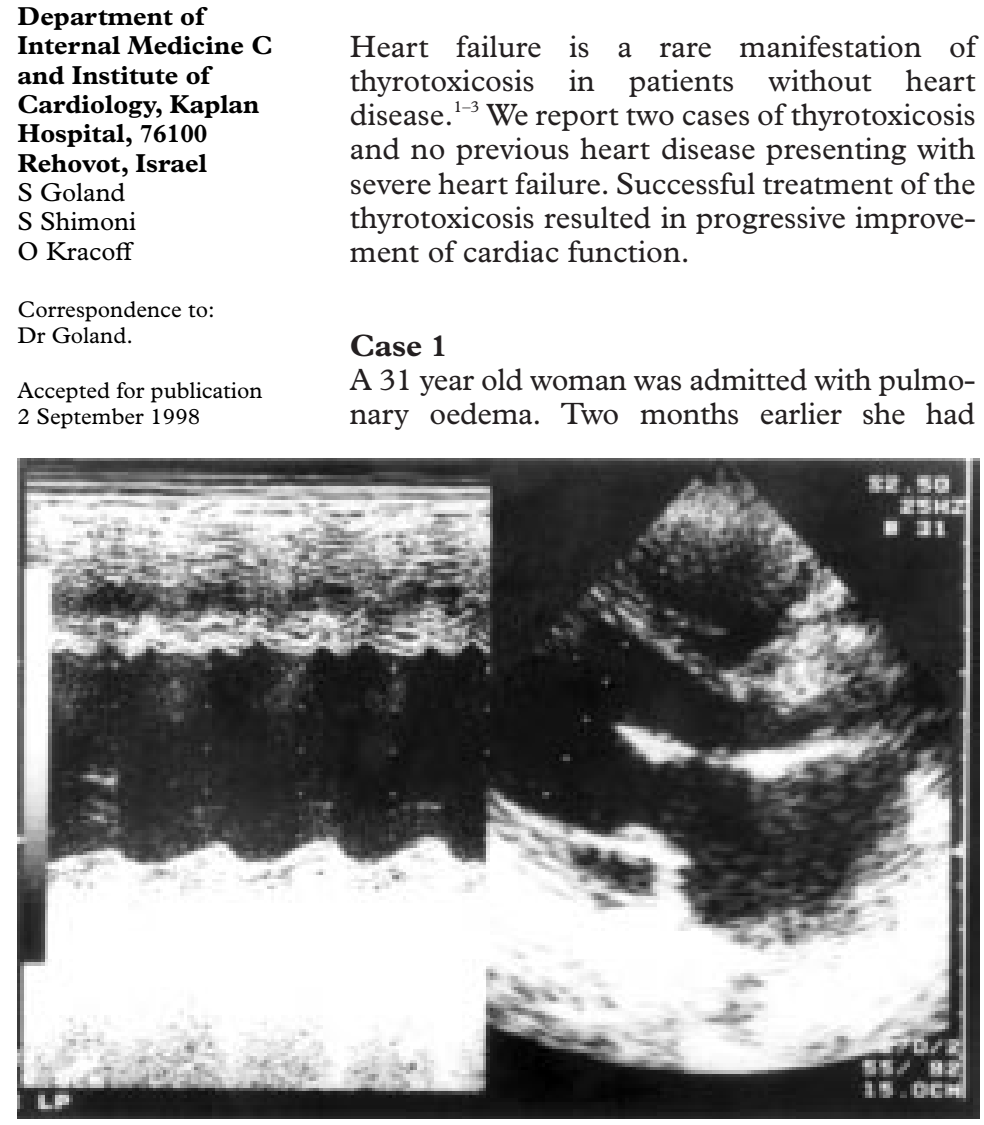

Figure 1 Parasternal long axis view (B mode and $M$ mode) showing reduced systolic left ventricular function. End diastolic diameter is $5.0 \mathrm{~cm}$; end systolic diameter is $4.2 \mathrm{~cm}$; estimated ejection fraction is $34 \%$.

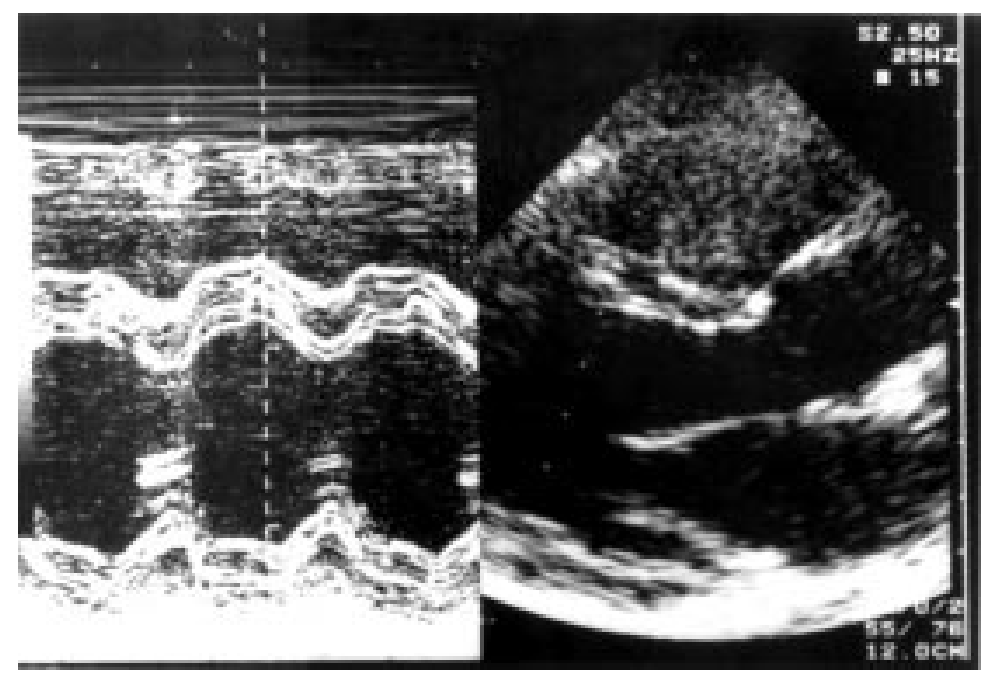

Figure 2 Parasternal long axis view showing normal left ventricular diameter and function. Estimated ejection fraction is $62 \%$. complained of fatigue, palpitation, and chest discomfort. She had no risk factors for coronary artery disease, had regular menses, and denied smoking or alcohol consumption. There were no symptoms of intercurrent infection.

On physical examination she looked very ill. Her heart rate was 120 beats/min and blood pressure $130 / 80 \mathrm{~mm} \mathrm{Hg}$. She had a small goiter and typical signs of thyrotoxicosis including warm, moist skin and a fine tremor. There was an audible S3 on cardiac auscultation. ECG showed sinus tachycardia, increased voltage, and ST depression in leads I, II, aVL, and V4-6.

Chest radiography was consistent with interstitial pulmonary oedema and moderate cardiomegaly. Echocardiography showed four chamber dilatation and depressed left ventricular (LV) function with an ejection fraction of 34\% (fig 1). Routine tests were normal as was erythrocyte sedimentation rate. She had mild anaemia (haemoglobin $115 \mathrm{~g} / \mathrm{l}$ ). Thyroid function tests were available later: thyroid stimulating hormone< $0.2 \mathrm{MU} / 1$ (normal range 0.3-4.5), total triiodothyronine $11 \mathrm{pmol} / 1$ (normal range $1.0-2.7$ ), and free thyroxine $60 \mathrm{nmol} / 1$ (normal range 8.9-24.3).

She was treated with intravenous frusemide and morphine. As thyroid function test results became available treatment with dexamethasone, propylthiouracil, and potassium iodide solution was added. A few hours later she developed atrial fibrillation, which converted to normal sinus rhythm after a short course of amiodarone treatment. She was discharged on oral propylthiouracil, propranolol, and captopril. One year later thyroid function tests were normal, she was free from any symptoms of heart failure, and continued only propylthiouracil treatment. Repeated echocardiography was normal (fig 2).

Case 2

A 46 year old man, who had a history of partial thyroidectomy because of Grave's disease, was admitted with pulmonary oedema. The clinical and laboratory results were typical of thyrotoxicosis. Following successful treatment of thyrotoxicosis with propylthiouracil and $\beta$ blockers his left ventricular ejection fraction increased from $34 \%$ to $50 \%$. He remains clinically well and euthyroid on maintenance propylthiouracil treatment.

\section{Discussion}

The association of thyrotoxicosis and cardiovascular morbidity is well established. There are several cardiac manifestations of thyrotoxi- 
cosis, including enlargement of the heart, atrial fibrillation, high output heart failure, hypertrophic cardiomyopathy, anginal syndrome without evidence of coronary artery disease, and sudden death. ${ }^{1-3}$ The high output state in thyrotoxicosis results from direct enhancement of heart rate and contractility by thyroid hormones, indirectly increasing blood volume and causing peripheral vasodilatation. High output heart failure develops despite increased cardiac performance. ${ }^{3}$ Low output heart failure is an extremely rare manifestation of thyrotoxicosis. There have been a few recent reports of dilated cardiomyopathy and congestive heart failure, especially in children and elderly patients with apathetic thyrotoxicosis. ${ }^{45}$ Most of the patients had complete or near complete recovery of cardiac function after treatment. ${ }^{45}$ However, Ebisawa et al reported that cardiomyopathy in patients with thyrotoxicosis may be irreversible even 15 years after successful treatment of their thyrotoxicosis. ${ }^{5}$ There has also been a report of sudden death in one young patient with thyrotoxicosis and dilated cardiomyopathy. ${ }^{6}$ There were no specific abnormalities in myocardial biopsy specimens taken from these patients.

We describe two young patient with thyrotoxicosis who developed dilated cardiomyopathy and pulmonary oedema as the first presenting symptom. Patients presenting with heart failure and dilated cardiomyopathy may have thyrotoxicosis as the underlying cause. Treatment of the thyrotoxicosis can restore left ventricular function. Awareness of this possible presentation of thyrotoxicosis may help identify patients with reversible dilated cardiomyopathy.

1 Woeber KA. Current concepts. N Engl f Med 1992;327:94-8.

2 Thyrotoxicosis and the heart: something old and something new [editorial]. I Clin Endocrin Metab 1993;77:332.

3 Polikar R, Burger AG, Scherrer U, et al. The thyroid and the heart. Circulation 1993;87:1435-41.

4 Umpierrez GE, Challapalli S, Patterson C. Congestive heart failure due to reversible cardiomyopathy in patients with hyperthyroidism. Am f Med Sci 1996;410:99-102.

5 Ebisawa K, Ikeda H, Maruta M. Irreversible cardiomyopathy due to thyrotoxicosis. Cardiology 1994;84:274.

6 Manger JA, Clark W, Allenby P. Congestive heart failure and sudden death in a young woman with thyrotoxicosis. West $\mathcal{F}$ Med 1988;149:86-91.

\section{SHORT CASES IN CARDIOLOGY}

Department of

Cardiology,

St Bartholemew's

Hospital, West

Smithfield, London

EC1A 7BE, UK

J P J Halcox

Correspondence to: Dr Halcox.

Accepted for publication 24 April 1998

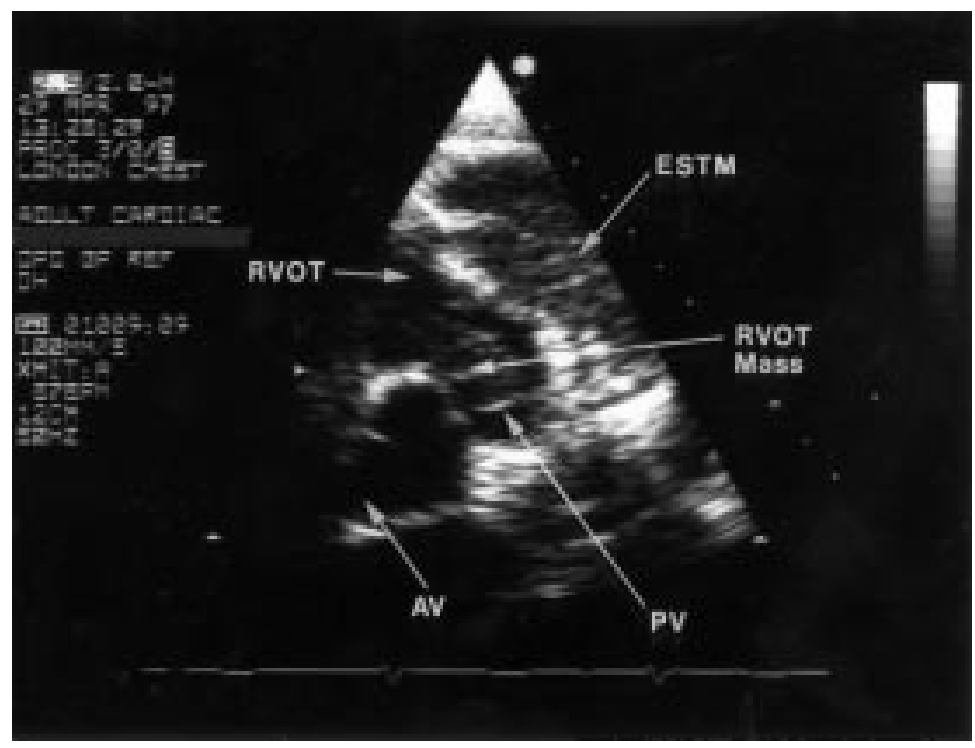

Figure 1 Parasternal short axis echocardiogram. RVOT, right ventricular outflow tract; PV, pulmonary valve; $A V$, aortic valve; ESTM, extracardiac soft tissue mass .

\author{
J P J Halcox
}

A 27 year old banker was referred by his general practitioner with constitutional symptoms and a murmur. He had a four month history of weight loss, lethargy and dry cough. On

\section{Non-surgical CHOP cures right ventricular outflow obstruction} 1) and ventricular outflow tract (RVOT) (fig 1) and colour flow mapping showed turbulent flow around its free edge. Flow velocity through the RVOT, assessed by continuous wave Doppler, was $4 \mathrm{~m} / \mathrm{s}$ estimating a pressure gradient of $64 \mathrm{~mm} \mathrm{Hg}$ (fig 2). The right ventricle was mildly dilated and an anterior extracardiac soft tissue mass was seen.

CT guided biopsy failed to get suitable tissue for analysis but a sample was obtained mediastinoscopically, histology of which confirmed sclerosing mediastinal non-Hodgkin's lymphoma.

The patient was treated with six cycles of cyclophosphamide, doxorubicin - formerly hydroxydaunorubicin, vincristine-oncovin, and prednisolone (CHOP) chemotherapy with excellent clinical and radiological response.

Repeated echocardiography one month after treatment showed complete resolution of the RVOT mass (fig 3) with normal spectral and colour Doppler assessment. 


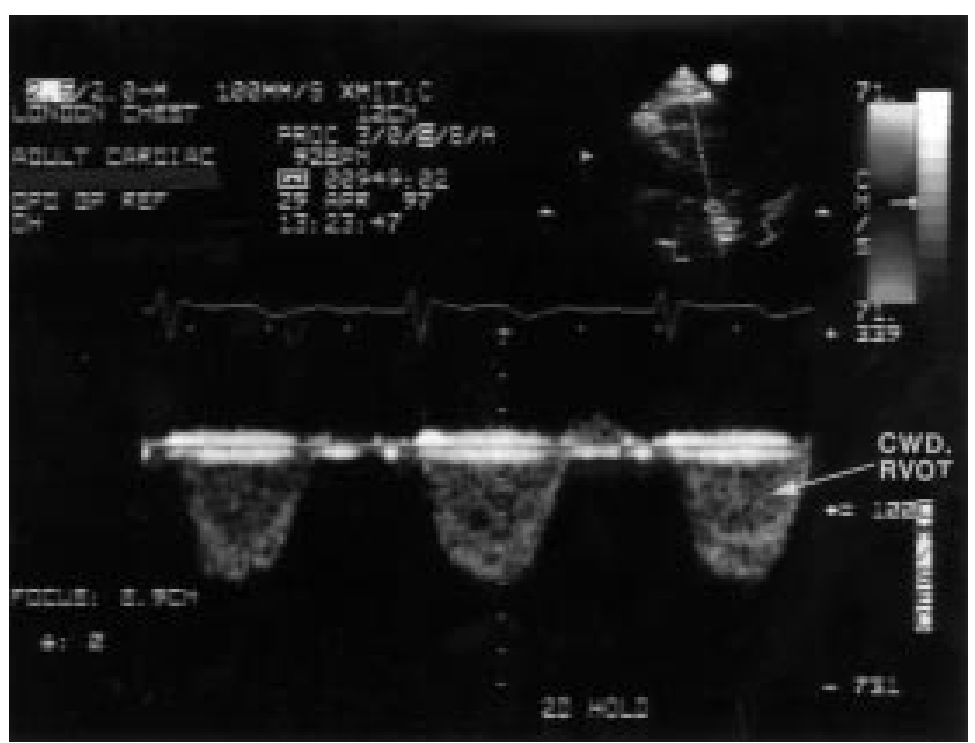

Figure 2 Continuous wave Doppler interrogation across the RVOT showing a peak velocity of $4 \mathrm{~m} / \mathrm{s}$ (pressure gradient of $64 \mathrm{~mm} \mathrm{Hg}$ ).

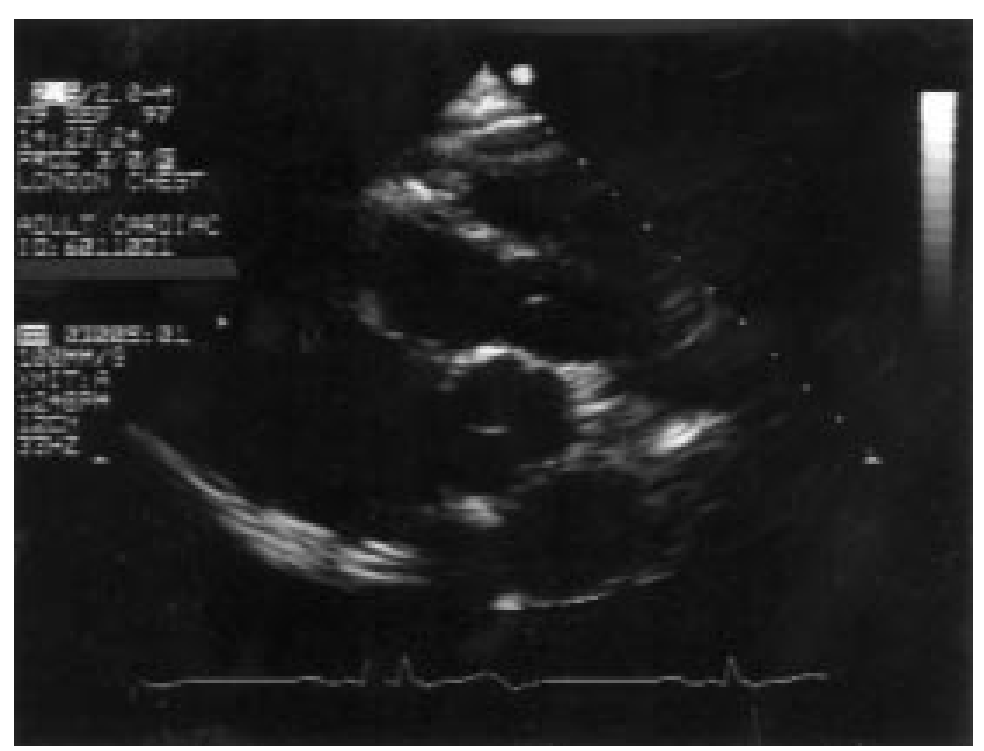

Figure 3 Repeat parasternal short axis scan one month after completion of chemotherapy showing resolution of the RVOT mass and reduction in extracardiac soft tissue shadowing.

\section{Discussion}

The differential diagnosis of an intracardiac mass includes vegetation, thrombus, and both primary and secondary neoplasm. Although the pericardium is the most usual site for cardiac secondary tumours, myocardial deposits are not infrequent. Lymphoma is noted as one of the more common tumours to spread to the myocardium..$^{1-3}$ The RVOT is a well recognised site of cardiac involvement with lymphoma. ${ }^{4}$

Our case illustrates the value of echocardiography in the assessment of such patients. The effect of an appropriate regimen of chemotherapy is clearly demonstrated with complete resolution of the tumour echocardiographically and radiologically.

I thank Ms D Harrington and Ms S Fergey for producing these informative images.

1 Lam KY, Dickens P, Chan AC. Tumours of the heart. A 20 year experience with a review of 12,485 consecutive autopsies. Arch Pathol Lab Med 1993;117:1027-31.

2 Abraham KP, Reddy V, Gatuso P. Neoplasms metastatic to the heart: review of 3314 consecutive autopsies. Am f Carthe heart: review of 3314 consel $1990 ; 3: 195-8$.

3 McDonnell PJ, Mann RB, Bulkley BH. Involvement of the heart by malignant lymphoma: a clinicopathologic study. heart by malignant lymp

4 Mills P, Wolfe C, Redwood D, et al. Non-invasive diagnosis of subpulmonary outflow tract obstruction. Br Heart $\mathcal{f}$ 1980;43:276-83.

5 Johnstone ID, Popple AW. Right ventricular outflow tract obstruction secondary to small intestinal lymphoma. $\mathrm{Br}$ Heart F 1980;43:593-6. 